\title{
Dendrimer Dipole Excitation: A New Mechanism for Terahertz Generation
}

\author{
Anis Rahman ${ }^{1 *}$, Aunik K. Rahman ${ }^{1}$ and Donald A Tomalia ${ }^{2}$ \\ ${ }^{1}$ Applied Research and Photonics, 470 Friendship Road, Suite 10, Harrisburg, PA 17111, USA \\ ${ }^{2}$ Nano Synthons LLC, 1200 N, Fancher Avenue, Mt. Pleasant, MI 48858, USA
}

\begin{abstract}
An electro-optic dendrimer (EO dendrimer) material has been designed for high power terahertz generation. An ordinary poly (amidoamine organosilicon) (PAMAMOS) dendrimer was modified via doping and poling to generate a high electro-optic coefficient nanomaterial. Measured EO coefficient varied from $\sim 130 \mathrm{pm} / \mathrm{V}$ at $633 \mathrm{~nm}$ to $\sim 90 \mathrm{pm} / \mathrm{V}$ at $1553 \mathrm{~nm}$. An emitter designed from this EO dendrimer generates milliwatts of continuous wave (CW) terahertz radiation (T-ray) when pumped by a CW laser of suitable wavelength. The mechanism termed as the dendrimer dipole excitation (DDE) works via excitation of the dipole population generated by the doping process. The doping protocol also generates a distribution of dipole moments, as opposed to fixed dipoles in the lattice of a crystalline material; thus, when excited by a suitable pump laser, these dipoles radiate a broadband frequency that range from $0.1 \mathrm{THz}$ to $\sim 30 \mathrm{THz}$. A terahertz time-domain spectrometer (TeraSpectra) was designed with this DDE terahertz source. As a test of the spectrometer functionality, a standard polyethylene calibration was conducted. It was found that TeraSpectra reproduces several known absorbance peaks of polyethylene. It also produces additional absorbance peaks not observed before. It is surmised that the ultra-high sensitivity of T-ray enables observation and discovery of additional absorbance peaks that are not visible via other spectroscopy such as visible, UV, FTIR or Raman.
\end{abstract}

Keywords: Electro-optic Dendrimer; Dipole Excitation; Terahertz Generation

\section{Introduction}

Currently, the so-called difference frequency generation (DFG) process [1] eliminates the use of a femto-second pulsed laser method for pulsed terahertz generation such as photo-conduction [2] and optical rectification [3]. Herein we now introduce a new and different mechanism; namely, dendrimer dipole excitation (DDE), as a strategy for tunable, continuous wave (CW) terahertz generation. Dendrimers are core-shell macromolecules possessing spherical molecular structure. The molecular size of a dendrimer is controlled by the number of concentric shells around the core; wherein, each shell is known as a generation $(G)$ [4]. Figure 1 shows the molecular structure of a dendrimer having three shells around the central core (G3). Each terminal group is capable of attaching two monovalent dopant molecules; thus the total number of probable dopants per dendrimer molecule is given by $2^{(G+2)}$. When the dendrimer is doped with a suitable chromophore, multiple dipole moments are created because of the distribution of charge centers within the dendrimer molecule. As can

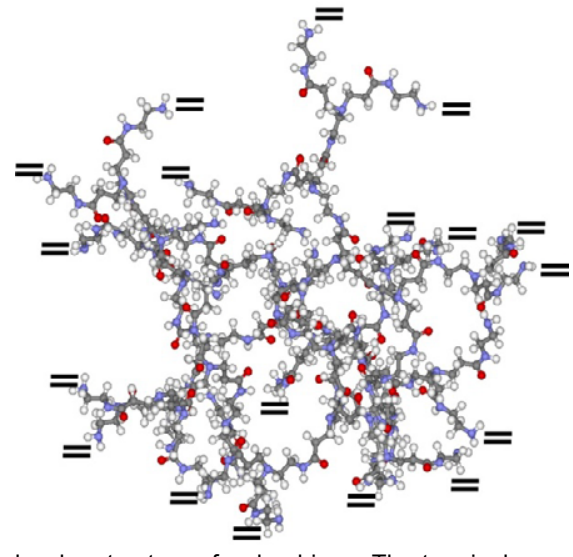

Figure 1: Molecular structure of a dendrimer. The terminal groups each have two sites available where dopant molecules may be attached. Thus a distribution of dipoles per molecule is possible via chromophore doping. be reasonably assumed from the molecular structure of a dendrimer, these dipoles have a distribution of dipole moment, because of the spacing distribution of the charge centers. As such, the equation for dipole moment, is $\mu=q l$ rewritten as, $\mu(x)=q l(x)$ where $\mu$ is the dipole moment, $q$ is the charge, and $l$ is the separation between the negative and positive charges forming the dipole. Figures $2 \mathrm{a}$ and $2 \mathrm{~b}$ shows the energy level diagram of difference frequency generation [5] and Figure 3 shows the schematic of possible energy levels in an electro-optic (EO) dendrimer resulting from chromophore induced dipole moment distribution created by the doping process. If this dipole distribution is excited by a suitable pump laser, a broadband emission is expected which falls in the terahertz range.

(b)

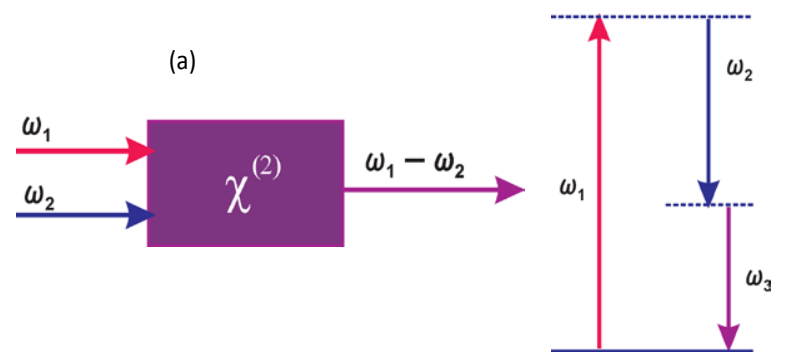

Figure 2: (a) Interaction geometry for difference frequency generation and (b) Energy-level diagram of difference frequency generation [4].

*Corresponding author: Anis Rahman, Applied Research and Photonics, 470 Friendship Road, Suite 10, Harrisburg, PA 1711, United States, Tel: 1717-2201003; E-mail: a.rahman@arphotonics.net

Received November 02, 2015; Accepted January 08, 2016; Published January 18,2016

Citation: Rahman A, Rahman AK, Tomalia DA (2016) Dendrimer Dipole Excitation A New Mechanism for Terahertz Generation. J Biosens Bioelectron 7: 196. doi:10.4172/2155-6210.1000196

Copyright: (c) 2016 Rahman A, et al. This is an open-access article distributed under the terms of the Creative Commons Attribution License, which permits unrestricted use, distribution, and reproduction in any medium, provided the original author and source are credited. 


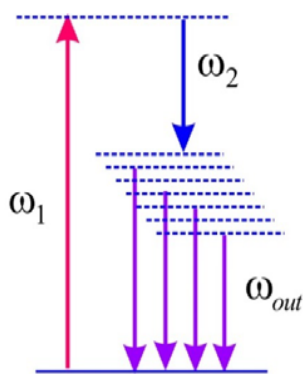

Figure 3: Possible energy level diagram in dendrimer molecule resulting from chromophore doping and poling. A distribution of dipole moments will create multiple quasi stable energy levels via which a broadband emission is energetically allowable.

In what follows, we describe an electro-optic dendrimer exhibiting higher electro-optic coefficient and higher second order susceptibility leading to a high power, wide broadband terahertz generation. Details of the doping and poling process have been described followed by terahertz generation and a time-domain spectrometer built around the DDE based terahertz source. Calibration of terahertz time-domain spectrometer via measurement of polyethylene is also described.

\section{Electro-optic dendrimer}

Electro-optic properties are important for many photonic applications that generally exploit the non-linear optical parameters such as electro-optic coefficient (EOC), and the second order susceptibility, $\chi^{(2)}$. These devices include terahertz emitter, optical modulator, and electro-optic sensors, among others. Manipulating the structural features of dendrimers allows a controlled enhancement of optical and electro-optic properties of these end-functionalized macromolecules. For instance, when chemically complexed (doped) with a high hyperpolarizability chromophore, a dendrimer's nonlinear optical (NLO) properties can be enhanced significantly. It is also known that dendrimers form a self-assembled multilayer on substrates whose thickness can be controlled by manipulating the dendrimer generation, surface and solution chemistry [6]. Because of their highly organized structure, dendrimers form high quality films that are suitable for lithographic fabrication of waveguide based photonic devices. Simultaneously dendrimers can be processed at relatively lower temperatures allowing integration with other functionality via a lithography process. For terahertz generation, high $\chi^{(2)}$ value plays an important role for both the up conversion and the bandwidth, as will be described later.

\section{Doping and poling of dendrimer}

Poly (amidoamine organosilicon) (PAMAMOS) dendrimers (Dendritech, Inc., Midland, Michigan) were used for the present investigation. PAMAMOS dendrimers are essentially a polyamidoamine (PAMAM) dendrimer [4] possessing end groups which are modified (3-acryloxypropyl)-trimethoxysilane (TMOS) that helps film forming property. For dendrimer doping, a few considerations are important in choosing a suitable chromophore. We strategized using commercially available chromophores to achieve a higher NLO property because this route can produce faster results than developing a new chromophore. PAMAMOS dendrimer has a functional group with relatively high affinity towards amine groups. Alizarin (1,2-dihydroxyanthraquinone, $\mathrm{C}_{14} \mathrm{H}_{8} \mathrm{O}_{4}$ ) has a suitable structure for complexing with dendrimer because its phenolic $\mathrm{OH}$ will interact with the PAMAMOS amine. However, the hydrogen bonding complex formation of a chromophore with the PAMAMOS requires an excess of amine to provide the proton transferred species. Therefore, a higher generation of PAMAMOS will allow a higher loading of a suitable chromophore if it satisfies bond forming conditions. We chose alizarin (Alfa Aeser) as the dopant because it is a non-linear chromophore with a large linear hyperpolarizability and also possesses absorption peaks in the IR region [7]. This is important for using dendrimers as a terahertz emitter. As a small molecule, alizarin not only can complex with the surface amine groups, but it can also fit in the interstitial spaces of a dendrimer molecule, thus help a uniform doping concentration giving raise to the diploe moment distribution outlined before. However, our goal is to lower doping concentration in dendrimer, so that the structural properties of the film are controlled by dendrimer itself while the electro-optic properties are influenced by the chromophore.

Thus, in a dendrimer, the chromophores may be attached to the end groups or be contained inside the molecular cavity because of the tertiary amine groups. Also, for effective doping, it is not essential for the chromophore molecule to complex (chelate) with the chelating sites in the PAMAMOS matrix, because, attractive force between the chelating sites and one of the functional groups in the chromophore should suffice for uniform doping. Consequently, dendrimers provide a suitable host for selective guest doping and enhancement of NLO properties.

Five different dendrimer films are shown in Figures $4 \mathrm{a}-4 \mathrm{e}$ for a visual inspection of refractive index variation indicated by color contrast. These films were formed by spin coating of dendrimer solutions on glass slides and then curing them on a hot plate in ambient atmosphere. For refractive index measurements, same solutions were spun on silicon wafers. Both glass slides and wafers were cured simultaneously with identical curing profile. Refractive indexes of the films on wafer were measured with a Metricon 2010 prism coupler. Films (a) - (d) are undoped dendrimer where the refractive index varies from 1.45 to 1.64 as a function of dendrimer generation. For electrooptic measurements, Alizarin doped dendrimer film were prepared by spin coating on silicon wafer.

Film (e) shows a film formed from dendrimer doped with 5\% alizarin before poling; this film has a refractive index $\sim 1.52$. All films were cured at a temperature between $150^{\circ} \mathrm{C}$ and $160^{\circ} \mathrm{C}$ using a 3 step ramp and soak protocol.

\section{Corona poling}

The objective of poling is to optimize the dipole alignment of the cured film. The principle is sketched in Figures 5a-5c. Like other polymer films, the value of non-zero $\chi^{(2)}$, and therefore, $r_{33}$, depends on the dipole orientation: $\chi^{(2)}=\langle n f \beta\rangle\left(\cos ^{3} \theta\right)$ where, $n$ is the density of dipole moments, $f$ is the local field factor, $\beta$ is the average hyperpolarizability and $\theta$ is the dipole alignment angle. To the first approximation, when the aligning field is static, the average orientation
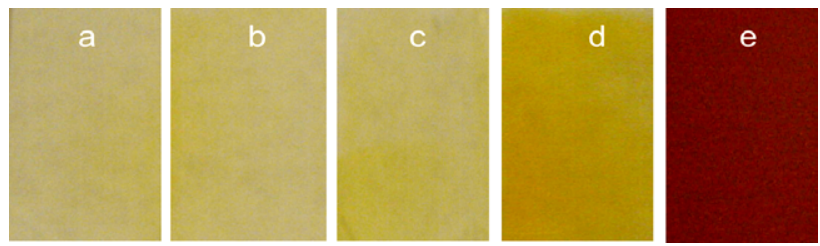

Figure 4: Dendrimer film on glass slides: (a)-(d) undoped dendrimer Refractive index varies from 1.45-1.64. (e) Chromophore doped dendrimer film: RI 1.52 
depends on the poling field strength. There are a number of poling techniques that have been deployed by other investigators. These are: solid or liquid contact electrode poling, all-optical poling, photo assisted poling and corona poling; all can break the molecular Centro symmetry to produce non-zero nonlinear effect. Here a corona poling was deployed because of the geometrical simplicity of the method and also to avoid complicacies associated with contact electrode poling [8]. For contact poling, electronic conduction within the polymer layers has been identified in many polymers. Even a very small conduction current within the film during poling will result in an incomplete (nonefficient) orientation of the dipole moments. Blum et al. [9] identified three regions of conduction as a function of applied field for Disperse Red 1 doped PMMA polymer where contact electrodes of ITO and gold were used on either sides of a waveguide. The authors found that for $\sim 1.44 \mu \mathrm{m}$ thick active layer cladded between two layers of SiOx [derived from poly(methyl siloxane)], an Ohmic conduction exists below $25 \mathrm{~V} / \mu \mathrm{m}$. Between 25 and $100 \mathrm{~V} / \mu \mathrm{m}$, conduction via Schottky thermionic emission is dominant while a further higher field may generate larger current via Fowler-Nordheim tunneling. We conducted a controlled corona poling in order to avoid those adverse side effects mentioned above. To our knowledge this is the first poling results for the PAMAMOS dendrimer film leading to higher EO coefficient. The sample configuration is shown in Figure 5; a high voltage needle electrode was placed $1 \mathrm{~cm}$ above the sample surface. Sample temperature was raised at a rate of $4^{\circ} \mathrm{C} / \mathrm{min}$ and held at $110 \pm 1^{\circ} \mathrm{C}$.

The degree of dipole orientation determines the pump- $\mathrm{THz}$ conversion efficiency while the concentration of the oriented dipole moments determines the total output power.

It is expected that as the alignment progresses, poling current will increase as a function of applied field [10,11]. The poling current should stabilize when maximum alignment is reached. Beyond this point, additional applied field would drive the poling current higher potentially leading to the phenomena observed by Blum et al. [9] and at a further higher field breakdown of the film may occur.

It was observed that the onset of producing a measurable current at $110^{\circ} \mathrm{C}$ is $>6 \mathrm{kV}$ of applied voltage for the configuration used. Both the temperature and the poling current were monitored during the entire period by an automated computer program to examine the poling condition. Voltage was increased at a step of $50 \mathrm{~V}$ and the resulting current was recorded. As seen from Figures $6 \mathrm{a}$ and $6 \mathrm{~b}$ the current increased as the voltage was raised above the threshold and stabilized

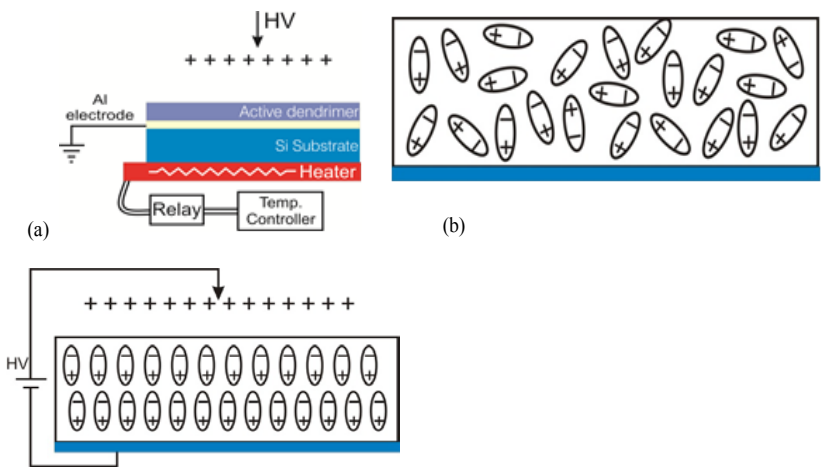

(c)

Figure 5: (a) Sample configuration for corona poling. (b) Sketch of dipole orientation in unpoled and (c) corona poled dendrimer film.
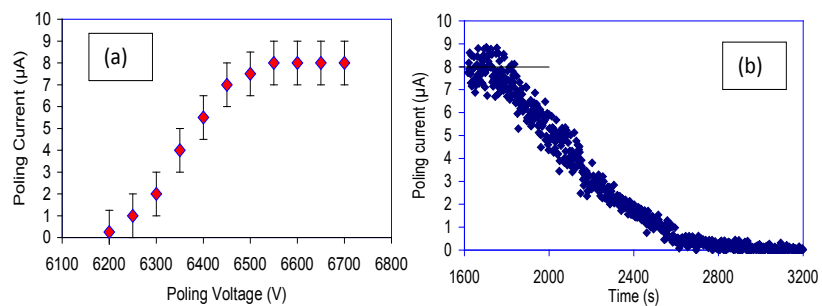

Figure 6: (a) Poling current of a chromophore doped dendrimer film. Current stabilizes at $\sim 8 \mu \mathrm{A}$ at $6700 \mathrm{~V}$ applied. The optimum poling voltage was between $6600 \mathrm{~V}$ and $6700 \mathrm{~V}$. (b) Current decayed at constant applied voltage of 6700 Volts as the temperature slowly reduced to room temperature.
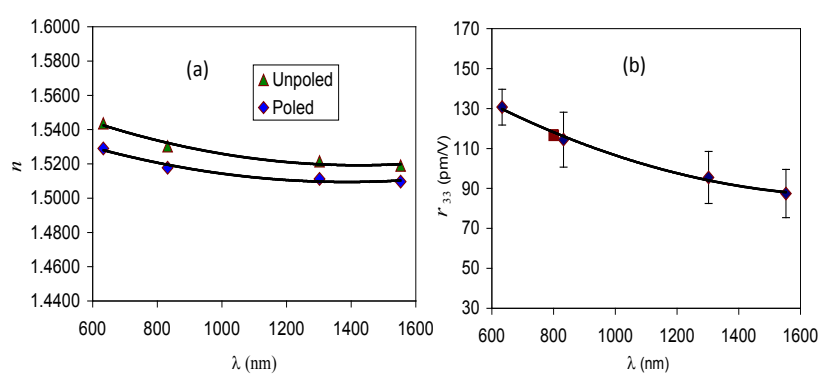

Figure 7: (a) Measured refractive index of poled and unpoled dendrimer film (b) Electro-optic coefficient of poled dendrimer film at room temperature; $r_{33}$ values were calculated from the linear Pockels effect, Eq. (1)

at $(8 \pm 1) \mu \mathrm{A}$ at an applied voltage of $(6600 \pm 100)$ Volts. Since the film thickness is $\sim 100 \mu \mathrm{m}$, this corresponds to the field strength of $\sim 6.6 \times$ $10^{5} \mathrm{~V} / \mathrm{cm}$. Current remained steady at the maximum applied voltage at a fixed temperature indicating an optimum alignment. Optimum stable poling current (at an applied voltage of 6700 Volts) was maintained for $\sim 30$ minutes to allow for the completion of orientation of the dipoles. At that point, while the high voltage was still being applied, the heater was turned off. As expected, the current subsided as the temperature slowly reached room temperature. This procedure ensures that the dipoles remain oriented and localized after poling.

\section{Electro-optic measurements}

Here the linear Pockels effect is used to deduce the $r_{33}$ parameter from measured refractive index change between unpoled and poled dendrimer film. The refractive indices were measured with a prism coupler (Metricon). The orientation distribution of the dipole moments of cured polymer film is isotropic. Hence the index ellipsoid is a sphere. After poling the isotropy is broken and the poled film becomes a uniaxial polar material with a changed refractive index. The difference in refractive indices, $\Delta n$, is related to the electro-optic coefficient via the poling field $E_{\mathrm{p}}$ as,

$$
|\Delta n|=\frac{1}{2} n^{3} r_{33} E_{p}
$$

Figure 7a shows the measured refractive index (RI) of both poled and unpoled films at room temperature as a function of wavelength. A systematic difference in refractive index has resulted due to poling. This difference is utilized in Eq. (1) to compute $r_{33}$; a value of $\sim 130$ $\mathrm{pm} / \mathrm{V}$ was obtained at $633 \mathrm{~nm}$ falling to $\sim 90 \mathrm{pm} / \mathrm{V}$ at $1553 \mathrm{~nm}$ (Figure $7 \mathrm{~b})$. This value is significantly higher than the inorganic crystalline materials, e.g., $\mathrm{LiNbO}_{3}$. Since the second order susceptibility is given by $\chi^{(2)} \propto \varepsilon^{2} r_{33}$, where $\varepsilon$ is the relative dielectric constant, a significantly 
high second order susceptibility is obtained for EO dendrimer.

\section{Terahertz emitter}

The difference frequency generation (DFG) is a stimulated emission process, described by a non-linear polarization [5],

$$
P\left(\omega_{1}-\omega_{2}\right)=2 \chi^{(2)} E_{1} E_{2}^{*},
$$

where, $E_{1}$ and $E_{2}$ are the field strengths and $\omega_{1}$ and $\omega_{2}$ are the frequencies of the input lasers. Here, the generated frequency is the difference of those of the applied pump-fields, thus is tunable. Difference-frequency generation can be described in terms of the photon energy-level diagram. In order to satisfy the conservation of energy requirements, a photon at the higher input frequency $\omega_{1}$ must be destroyed and a photon at the lower input frequency $\omega_{2}$ must be created for every photon that is created at the difference-frequency $\omega_{3}=\omega_{1}-\omega_{2}$. The lower-frequency field in effect is amplified by the process of difference-frequency generation, thus, it is a stimulated amplification process. According to the photon energy-level description of the DFG, the atom (molecule) first absorbs a photon of frequency $\omega_{1}$ and jumps to the highest virtual (unstable) level. This level decays by a two-photon emission process that is stimulated by the presence of the $\omega_{2}$ field. However, two-photon emission can occur even if $\omega_{2}$ was not applied. Therefore, $\omega_{2}$ input field acts similar to an idler frequency of a parametric oscillator that is in effect filtered out (Figure 8).

For the input pump, two fiber-coupled single-mode diode lasers beam were overlapped in a beam-combiner. Two Amrel PPS-10710 power supplies were used to provide the input current at fixed bias to the diode lasers. Figure 8 shows the experimental setup to measure the average $(\mathrm{CW})$ terahertz power from DFG. Here, overlapped beam was normally incident on the dendrimer emitter. The detector (Astral AC2500) was fitted with a spectral filter that truncates wavelength down to $1500 \mathrm{~nm}$ and also a $3.3 \mathrm{~mm}$ thick polyethylene filter such that no visible light or IR can enter the detector. Figure 9 shows the measured DFG average power as a function of the total pump power from diode1+diode2. As seen from Figure 9, $3.4 \mathrm{~mW}$ of terahertz power was obtained from the dendrimer emitter at a combined pump power of $5.5 \mathrm{~W}$. To our knowledge, this is the highest average $\mathrm{CW}$ power from any compact terahertz sources, either polymeric or inorganic. This is easily tunable and higher terahertz power may be obtained by tuning the pump power. The center wavelength of diode 1 is $\lambda 1=975 \mathrm{~nm}$, and the center wavelength of diode 2 is $\lambda 2=940 \mathrm{~nm}$. Therefore, it is expected that the source of Experimental setup for terahertz measurement via difference-frequency generation should yield a broadband terahertz radiation,

$$
v_{T H z} \propto v_{1}-v_{2} \sim 30 \mathrm{THz} .
$$

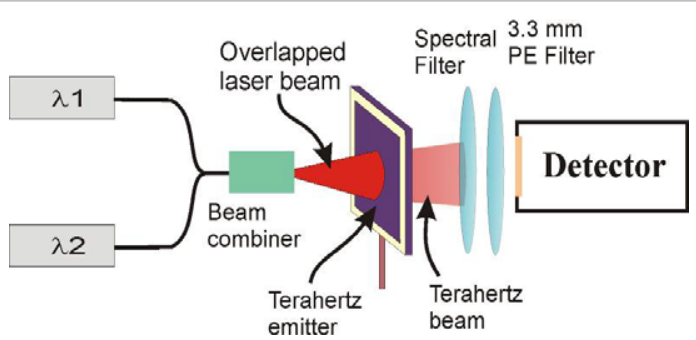

Figure 8: Experimental setup for terahertz measurement via differencefrequency generation.

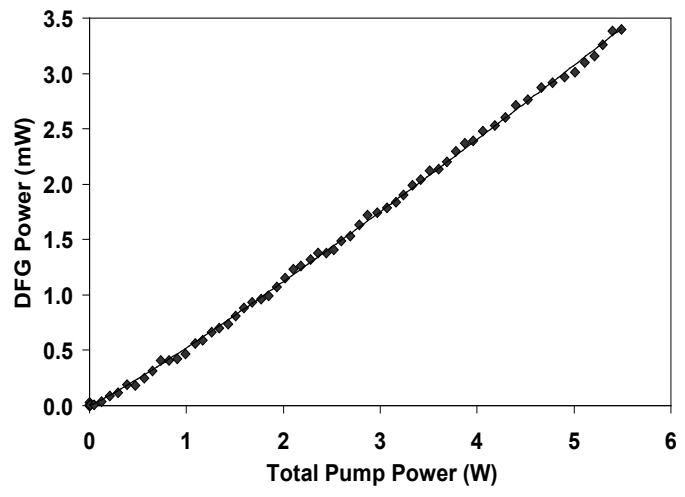

Figure 9: Measured DFG average power vs. total pump power from (diode1+diode2).

\section{Terahertz spectrometer}

A terahertz time-domain spectrometer (TeraSpectra) was designed around the DDE based source; details of its design and functionality were described elsewhere [12]. Briefly, the pump laser beam is split into two arms of $80: 20$ power ratios. The 80 -arm is used as the pump beam that passes through an optical delay line before being incident on the dendrimer emitter, while the 20 -arm (probe beam) remains stationary. This is known as the so-called pump-probe technique. An interferogram is generated in the time-domain when the probe beam is scanned by the terahertz beam whose intensity distribution is captured by a pair of detectors each oriented in mutually orthogonal directions. In order to increase the dynamic reserve of the spectrometer it is important that the ratio of the pump-arm and the probe arm should remain fixed throughout the operation. Since the TeraSpectra is a time-domain spectrometer where the sample response resulting from the molecular interaction with terahertz radiation is recorded in timedomain, the terahertz spectrum, i.e., the observable as a function of frequency or wavenumbers, is obtained by means of Fourier transform of the time-domain signal. Thus, calibration of the spectrometer involves reproducing known absorption peaks of standard materials to ensure measurement accuracies in this spectral region. However, it is found that TeraSpectra not only reproduces the known peaks, but also gives additional peaks - owing to its higher sensitivity. The theoretical justification of these additional peaks may or may not be readily available. The origin of the additional peaks is due to the fact that unlike other spectrometer techniques such as, IR, UV/Vis, Raman, etc., terahertz is sensitive to all kinds of resonances that may be present in a given molecule, such as, translational, rotational, vibrational, torsional, etc., [13] and also conformational. These additional peaks are thus expected, and therefore, justifies the emergence of a new spectrometer where indeed additional information is generated that are not available from its predecessors.

\section{Calibration}

In this section we describe measurements with an aim to compare data obtained from ARP's TeraSpectra system with those published in the contemporary literature or elsewhere. A standard polyethylene card was used for measurements [14]. The experimental setup is shown in Figure 10. Following procedure was used:

a) The TeraSpectra was stabilized overnight.

b) A time-domain signal ( $\mathrm{THz}$ pulse or interferogram) was generated for the empty spectrometer. 


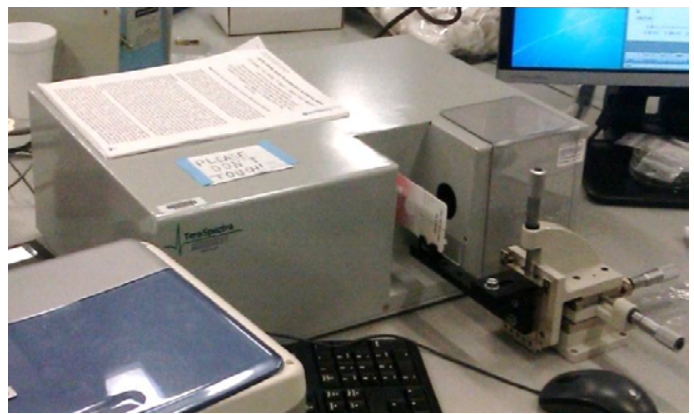

Figure 10: A polyethylene card mounted on the xyz-stage; positioned for maximum transmission. The setup is located at the Materials Research Institute, The Pennsylvania State University, University Park, PA 16802.

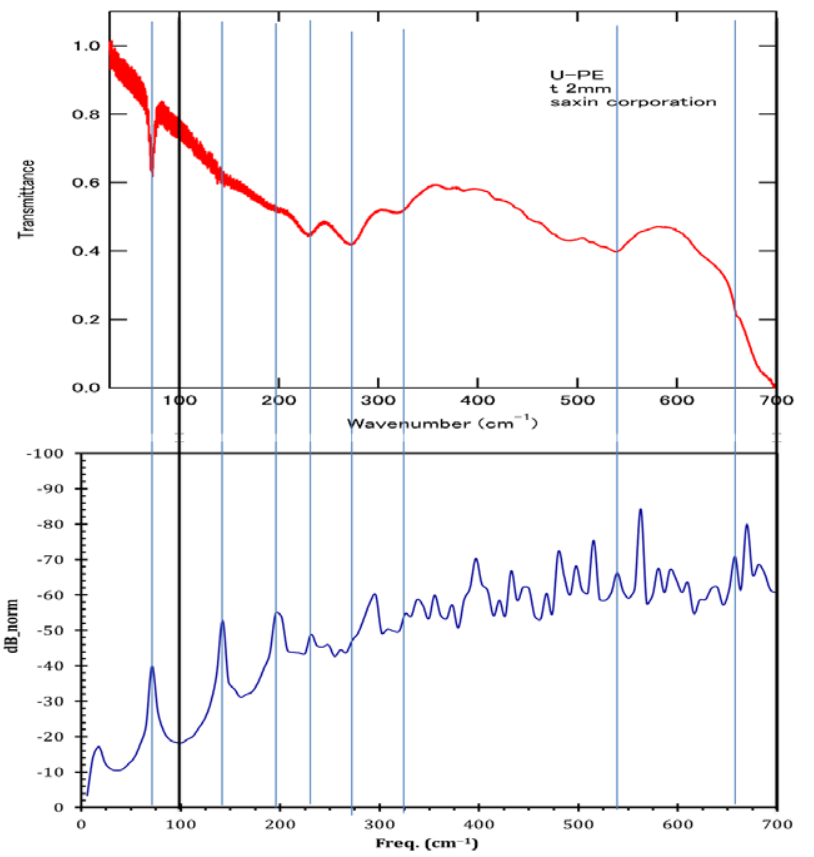

Figure 11: Comparison of PE spectrum generated by TeraSpectra with that reported by $\mathrm{THZ}$ database [13]. The matching peaks indicated by the blue lines are in good agreement between the two.

c) The PE card was positioned in the beam path for maximum transmission (Figure 10); no further change in any settings of the spectrometer.

d) $\mathrm{THz}$ pulse was recorded for the PE card.

e) Fourier transform was performed.

f) Absorbance spectrum was analyzed for matching peaks (Figure 11).

Figure 11 exhibits plots of $\mathrm{PE}$ spectrum reported by the $\mathrm{THz}$ database [13] (top plot) along with the close-up of the PE spectrum generated by TeraSpectra (bottom plot). As seen from Figure 11, there is a one-to-one match of several absorbance peaks. Figure 12 shows the Fourier transform absorbance spectrum up to $30 \mathrm{THz}$. As seen from Figure 12, there are many more new absorption peaks visible compared to those observed from previous methods. The origin of the new peaks needs to be further analyzed. Thus, it was found that the

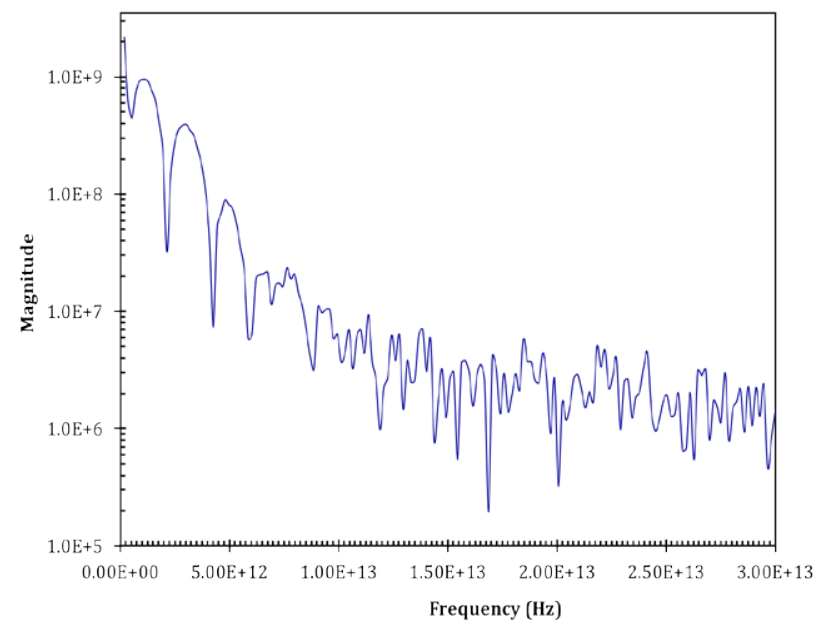

Figure 12: Polyethylene spectrum generated by the Tera Spectra.

\begin{tabular}{|c|c|c|}
\hline Source & Reported (1/cm) & TeraSpectra (1/cm) \\
\hline Sigma-Aldrich & 70.8 & 71 \\
\hline Free Snell [14] & 723.3 & 722.5 \\
\cline { 2 - 3 } & 749.5 & 746.2 \\
\hline
\end{tabular}

Table 1: Comparison of reported absorbance peaks with teraspectra.

spectrum obtained from TeraSpectra reproduced absorbance peaks known from other methods [13] and also discovered new peaks not visible from other methods. A few closely matching peaks' values are shown in Table 1.

\section{Summary and Conclusions}

This paper describes some details of high EO coefficient dendrimer film preparation and characterization. Here a commercially available dendrimer was modified via doping and poling to obtain a higher electro-optic coefficient, thus creating an electro-optic (active) dendrimer nano material. The difference in the refractive index of the poled and unpoled EO dendrimer utilized via the Pockel's law [Equation (1)] yields an EO coefficient of $\sim 130 \mathrm{pm} / \mathrm{V}$ at $633 \mathrm{~nm}$ and $\sim 90 \mathrm{pm} / \mathrm{V}$ at $1553 \mathrm{~nm}$. Terahertz generation from the said EO dendrimer via a mechanism termed as the dendrimer dipole excitation (DDE) mode is also described. A spectrometer designed around this terahertz generator has been used to measure standard polyethylene samples. The time-domain signal (or the interferogram) was analyzed for Fourier transform absorbance spectrum. It was found that the measured spectrum reproduces known peaks of polyethylene. Additionally many new peaks are visible that were not seen before. The origin of these additional peaks are assumed to be due to the fact that terahertz radiation is sensitive to all different kind of resonances present in a molecule such as translational, vibrational, rotational, torsional and conformational states. Hence the terahertz interaction with the material is expected to produce additional absorbance peaks that are not visible by less sensitive techniques. Observation of these additional peaks also strengthens the emergence of this new kind of approach for time-domain spectroscopy. Additional investigations are to be conducted to find any difficulties with this technique and also for exploring new physical phenomena.

\section{References}

1. Simon U, Miller CE, Bradley CC, Hulet RG, Curl RF, et al. (1993) Difference- 
frequency generation in AgGaS2 by use of single-mode diode-laser pump sources. Optics Letters 18: 1062-1064.

2. Auston DH, Glass AM, Ballman AA (1972) Optical Rectification by Impurities in Polar Crystals. Phys Rev Lett 28: 897-898.

3. Chang G, Divin CJ, Liu CH, Williamson SL, Galvanauskas A (2006) Power scalable compact $\mathrm{THz}$ system based on an ultrafast $\mathrm{Yb}$-doped fiber amplifier. Optics Express 14: 7909-7913.

4. Tomalia DA (2004) Birth of a new macromolecular architecture: Dendrimers as quantized building blocks for nanoscale synthetic organic chemistry. Aldrichimica Acta 37: 39-57.

5. Boyd RW (1992) Nonlinear Optics ( $2^{\text {nd }}$ edn.). Academic Press, New York.

6. Rahman KMA, Durning CJ, Turro NJ, Tomalia DA (2000) Adsorption of Poly(amidoamine) Dendrimers on Gold. Langmuir 16: 10154-10160.

7. Cyranski MK, Jamroz MH, Rygula A, Dobrowolski JC, Dobrzycki L, et al. (2012) On two alizarin polymorphs. Cryst Eng Comm 14: 3667-3676.
8. Herman WN, Cline JA (1998) Chielectric relaxation: Chromophore dynamics in an azo-dye-doped polymer. J Opt Soc Am B 15: 351-358.

9. Blum R, Sprave M, Sablotny J, Eich M (1998) High-electric-field poling of nonlinear optical polymers. J Opt Soc Am B 15: 318-328.

10. Gadret G, Kajzar F, Raimond P (1991) Nonlinear Optical Properties of Poled Polymers. In Nonlinear Optical Properties of Organic Materials IV SPIE 1560: 226-237.

11. Teng CC, Man HT (1990) Simple reflection technique for measuring the electrooptic coefficient of poled polymer. Appl Phys Lett 56: 1734-1736.

12. Rahman A (2011) Dendrimer Based Terahertz Time-Domain Spectroscopy and Applications in Molecular Characterization. Journal of Molecular Structure 1006: 59-65.

13. Riken (2011) Terahertz light source research team.

14. Jaffer A (2012) FreeSnell: Polyethylene. 